$\beta$ Scorpii pr.

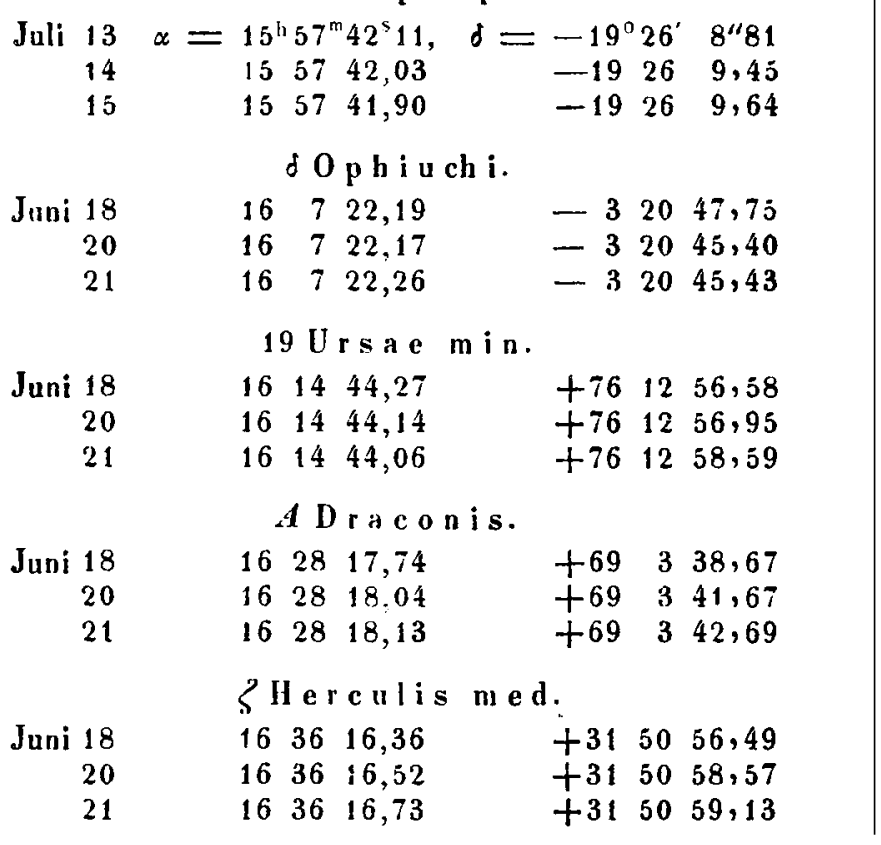

Gr. 2377 .

Aug. $9 \alpha=16^{\mathrm{h}} 42^{\mathrm{m}} 46^{\mathrm{s}} 80, \delta=+57^{\circ} 1^{\prime} 42^{\prime \prime} 13$ $k 0 \mathrm{phiuchi}$.

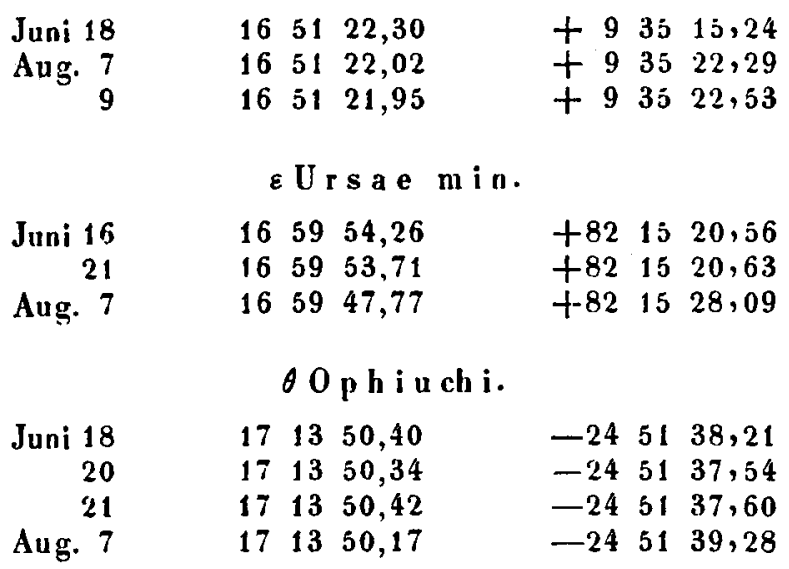

Gabriel Strasser, k. k. Professor und Astronom.

\title{
Ueber die scheinbaren Ausgangspunkte der Meteorbahnen.
}

Von dem Director der königlichen Sternwarte in Athen, Herrn $J$. F. Julius Schmidt.

Seit der letzten Erscheinung der Novembermeteore im Jabre 1866 hat eine Reibe von neuen Thatsachen und damit verbundenen Hypothesen ein nicht gewöhnliches Interesse erregt. Es hat sich anch gezeigt, dass ein Theil der Schlisse noch nicht genügend durch Beobachtungen gestützt werden knnute, und dass sehr vicle Facta und Erfabrungen, die eioige wenige ausdauernde Beobachter besitzen, mehrfach unbekannt geblieben sind, wenn sie auch seit vielen Jahren veröffentlicht wurden. Mir schien es nun zeitgemäss, einen Theil solcher Thatsachen, so weit sie mir in bequemer Weise aus meinen Papieren zugänglich waren, an's Licht zu ziehen, in der Hoffnung, dass diese und andere nach und nach den ganzen Umfang des complicirten Meteorphänomens denjenigen bekannt werden lassen, die nicht selbst lange Jahre hindurch Beobachter waren, und um gelegentlich einzelnen vielleicht zu raschen Schlussfolgerungen vorzubeuged. Diesmal wïhle ich die Betrachtung der Radiation der Meteore. d. h. der Punkte des Himmels, aus denen, gemäss der Perspective an der Sphäre, die unter sich ganz oder nahe parallelen Bahnen der Meteore auszugehen scheinen. Die Punkte im Löwen und Perseus sind seit. Langem bekannt; die meisten andern nördlichen Radiationen sind seit dem Anfange der vierziger Jahre von Professor $\boldsymbol{E}$. Ileis, in der Hauptsache nach seinen eigenen, zum Theil nach meinen Beobachtungen, entdeckt und genauer numerisch erörtert worden. Die bis 1852 erlanglen $t$... findet man in meiner „Resuitaten aus zebnjährigen Beobachtungen der Sternschnuppen" pag. 145 besprochen. Seit jener Zeit hat Heis andere Punkte der Radiation am nördlichen Himmel nachgewiesen, über welche der bis jefzt wichtigste Bericht sich in 1642 der Astron. Nachr. findet. Von 1859 an habe ich zu Athen viele südliche Divergenzpunkte der Meteorbahnen aufgefunden, und einige annähernd numerisch bestimnt, ohne indessen, wegen des Mangels ciner passeuden Himmelskugel im Stande zu sein, genaue Untersuchungen anzustellen. Viele hundert Babnen babe ich nicht verzeichnet, sondern nur angemerkt, zu welcher Radiation sie gehörten. Auch obne Zeichnung oder Construction weiss der erfahrene Beobachter darüber zu entscheiden, und zwar durch den blossen Anblick der Bahnen, die er sich rückwärts verlängert denkt, und deren so entstandene Durchschniltspunkte er notirt. Dahei werden die zweifelbaften Fälle vorläufig nicht berücksichtigt, und deren sind es oft viele. Solche Fälle treten ein, wenn sich Meteore in einem grössten Kreise bewegen, der ganz oder nahe zwei oder mehrere Radiationspunkte berührt. Unter den Athener Beobachtungen finde ich nun bei Gelegenheit der Bestimmung der stündlichen Häufigkeit der Meteore zahlreiche Angaben über diejenigen Ausgangspunkte der Bahnen, die in einer und derselb e Nacht vertreten waren. 
Gegenwärtig bin ich zwar nicht in der Lage, für jeden Tag alle. angedeuteten. Radiationen anzugelien; ich muss mich darauf leschränken, das mir jelzt zugängliche Material auf ubersichtliche Weise darzustellen.

Demnach wird man erkennen, dass die meisten Dirergenzpunkte viele Monate bindurch Meteore aussenden; dass im Sinne der Häufigkeit der Rang jener Punkte oft wechselt, nicht nur im Laufe der Wochen oder der Monate, sondern auch im Verlanfe ciner und derselthen Nacht. Man licmerkt, dass die Lage solches Ausgangspunultes gegen den Horizont und gegen den Meridian nicht gleichoülig für die Sichlbarkeit (Häufigkeil) Jer Meteore sei, und dass die Vorstellung, „es hewege sich ein isolirter Schwarm von Meteoren allein in eiper Bahn um die Sonne," nur ausnahmsweise, vielleicht" aher niemals in Wirklichkeit siattfude. Der Wahrheit näher wird man kommen, wenn man, z. B. rücksichtlich des Novemberstromes, sich vorstellt, dass zwar ein grosser Hauptstrom existire, der zu Zeiten an $13^{\text {ten }}$ November aus jetzt bekannten Ursachen gesehen wird; dass aher in dem ganzen Unfange jener Bahn andere, isolirt hinzieheude Meteore rorhanden sind. Man findet endlich, dass zur Zeit der grössten Häufigkeit der Metenre auch die ineisten Radiationspunkte gleichzeitig auftreten. Dies, und vieles Andere,

t bis jetzt allgemein nie genügend hekannt geworden; wenigen seitherigen Beobachtern konnten aher derartige

. radthisse nicht lange verborgen bleiben. In einer mir vorliegenden Tafel finden sich nach eigenen $26 \mathrm{jährigen} \mathrm{Beob-}$ achtungen, für die meisten Nächte, die jedesmal ermittelten Radiationspunkte aufgezeichnet; auf einer andern habe ich alle Angahen construirt, so dass die verschiedenen Radiationen als Abscissen, die Tage als Ordiuaten gelten. So entstand ein sehr anschauliches Bild, wie in Laufe des Jahres die verschiedenen Radiationen nach und nach auftreten, und hinsichllich der Häufigkeit ihres Auftretens ihren Rang wechseln. Beide Tafeln werde ich jetzt nicht mittheilen, weil sie allzuviel Raum beanspruchen; sie verdimen es auch nicht wegen ihrer mir svohlhekannten Unvollstäudigkeit. An ihrer Stelle will ich ein passendes Bruchstück, und eine Uelersichtstafel von geringer Ausdehnung hersetzen. lch wähle Fragmente aus einigen Monaten, wobei ïberall die Alloner Berbachtungen sebr das Uebergewicht haben. Nehen den Datum findet man den jerlesnal bemerkten Radiationspunkt, bezeichnet nach demjenigen Sternbilde, worin er sich befindet. Wenn es also heisst: Fehr. 28 Leo, Scorpius (1865), so will dies sagen: „186.5 Fehr. 28 gab es Meteore, die ihren Ausgaugspunkt (vermöge der rückwäirts verlängerten Bahnen) in Bilde des Lö we n und in Bilde des Scorpiones hatten." Der scheinbare Ort jener Metenre, also die Sterubilder, welche sie durchzogen, ist für unscre jetzige Betrachtung ganz gleichgültig. Die Jahreszahlen gebe ich in abgekürzter Form, z. B. 60 anstatt 1860 . Keinesweges sind jedesmal die angegebenen Radiationen die allein sichtbaren gewesen. Ich gebe nur, was ich notirt finde.

$$
\text { B e is piel. }
$$

Febr. 30 Can. Minor. oder Hydra. 39.

$$
\begin{aligned}
& =21 \text { Pol. Leo. } 57 . \\
& =23 \text { Leo. Can. Maj. } 57 . \\
& =25 \text { Leo. Geniui. } 57 . \\
& =26 \text { Leo. } 67 . \\
& =27 \text { Leo. } 57 . \\
& =28 \text { Leo, Scorpius. 65. Leo, Auriga. } 67 .
\end{aligned}
$$

April 19 Leo. 66.

$=20$ Lyra. 62 .

$=22$ I.yra, I.eo. 62.

$=23$ Leo. 59. Draco, Lyra, Leo. 62. Perseus. 66.

$=25$ Len. 65 .

$=27$ Leo. 67 .

$=28$ Caput. Serpentis. 65.

Juni 1 Sagittarius, Aquila, Cygnus, Pol. 64. Lyra. 67.

- 2 Sagittarius. 64. Ophiuchus, Aquila, Lyra. 67.

$=3$ Nördliche Rad. und Scorpius. 64. Lyra, Aquila, Pegasus, Cygnus. 67.

4 Scorpius. 64. Pegasus, Cygnus, Aquila. 67.

5 Scorpius. 64. Scorpius. 66. Lyra, Aquila, Pegasus. 67.

6 Pol. 64. Lyra, Ophiuchus. 67.

7 Scorpius, Pol., Cygnus. 64.

Juli 22 Nördliche u. südl. Rad. 59. Aquila, Lyra, Cygnus, Aquarius, Caper. 65.

$=23$ Pegasus, Draco, Cetus und andere südl. Rad. 59. Cassiopea, Aquila, Aquarius, Caper, Cetus. 65.

= 24 Südl. Rad. 59. Cefus, Phönix, Caper. 60. Perseus, Aquila, Cetus, Caper, Sagittarius u. a. südliche Punkte. 67. Aquarius, Caper, Cygnus, Aquila. 65.

$=25$ Aquila, Cygnus, Perseus. Cassiopea, Pol. u. südl. Rad. 59, Aquila, Aqnarius. 64. Caper, Aquarius, Aquila, Cassiopea, Lyra, Pisces, Cetus, Lepus, Perseus. 65.

$=26$ Aquila, Cygnus, Cassiopea und süll. Punkte. 59. Aquila, Pol., Cassiopea, Cygnus, Sagittarius. 64. Pol., Lyra, Cyenus, Cassiopea, Aquila, Caper, Aquarins, Pisces, Cetus. 65.

$=27$ Nördl. u. südl. Rad. 59. Sagitfarius, Caper, Aquarius, Aquila, Draco, Cygnus, Cetus, Cassiopea. 65.

$=28$ Cygnus, Lyra, Aquila, Caper. 59. Nördl. u. südl. Punkte (61), Pol., Sagittarius, Aquila, Caper, Pisces, Cetus, Perseus. 64. Sagiftarius, Aquila, Caper, Aquarius, Lym, Cygnus, Cassinpen, Perseus, Cetus. 65. 
Aug.10 Perseus, Cassiopea (1841-1866). Sehr viele Rad. 59. Cassiopea, Persens, Cygnus, Aquila, Lyra, Auriga, Taurus, Leo. 64. Cassiopea, Persens, Cygnus, Caper. 65. Cassiopea, Cygnus, Aquarius, Sagittarius, Cetus, Aries (66).

Sept. 4 Perseus, Cassiopea, Cygrnus und südl, Punkie. 61. Cygnus, Perseus, Cassiopea, Aquarius, Caper, Draco.64.

Oct. 19 Orion. 62. Taurus, Perseus, Leo. 63. Cassiopea, Perseus, Aquarius, Pol. 64. Perseus, Cassiopea, Cetus, Aquarius, Leo, Genini, Auriga, Taurus, Eridanus. 65.

Nov. 14 Pol., Cassiopea, Leo. 57. Perseus, Pol., Aquila, Leo, Gemini, Canis Min. Hydra. 63. Leo, Perseus, Cass. 66.
Dec. 12 Perseus, Gemini, Taurus, Eridanus. 63. Pol., Perseus, Gemini, Columba, Hydra, Leo. 66.

In der folgenden Tafel wird angegehen, wie viele Radiationspunkte wenigstens in jeder Nacht vertreten waren, alles rornehnlich nach Athener Beobachtungen. In Wahrheit sind die Zahlwerthe meist grösser; doch sind einzelne Ausnahmen vorhanden. Bis jetzt habe ich kein Bedenken, diese Werthe als Minina der Häufigkeit des Aufretens der Ausgangspunkte zu bezeichnen. Man erkennt, dass sie nahe mit den Zahlen der stündlicben Häufigkeit der Meteore coïncidiren. Die stündliche Anzahl der Yeteore, für den Gesichtskreis e ines Beobachters, nenne ich $z$. Zahlreiche Angaben dieser Art habe ich in Heis' Wocherischrift mebrfach mitgetheilt.

Mininalzablder in jeder Nacht auftretenden Radiationspunkte.

\begin{tabular}{|c|c|c|c|c|c|c|c|c|c|c|c|c|}
\hline$\underbrace{\text { Datum. }}$ & $\underbrace{\text { Jan. }}$ & $\underbrace{\text { Febr. }}$ & $\underbrace{\text { März. }}$ & $\underbrace{\text { April. }}$ & $\underbrace{\text { Mai. }}$ & $\underbrace{\text { Juni. }}$ & $\underbrace{\text { Juli. }}$ & $\underbrace{\text { Aug. }}$ & $\underbrace{\text { Sept. }}$ & $\underbrace{\text { Oct. }}$ & $\underbrace{\text { Nov. }}$ & $\underbrace{\text { Dec. }}$ \\
\hline 1 & - & - & - & - & - & 5 & 1 & 6 & 5 & 4 & 5 & 3 \\
\hline 2 & 1 & 1 & - & - & - & 4 & 4 & 4 & 7 & 3 & 6 & 4 \\
\hline 3 & - & 1 & - & - & 2 & 5 & 6 & 8 & 5 & - & 9 & 2 \\
\hline 4 & - & 1 & - & 1 & - & 4 & 3 & 9 & 6 & 1 & 5 & 4 \\
\hline 5 & 1 & - & 1 & - & - & 4 & 4 & 7 & 2 & - & 7 & 5 \\
\hline 6 & - & 1 & - & - & 1 & 3 & 2 & 10 & 3 & 1 & 7 & 4 \\
\hline 7 & 1 & - & 2 & - & - & 3 & 2 & 8 & 3 & - & 3 & 2 \\
\hline 8 & - & - & 2 & - & - & j & 2 & 11 & 9 & 7 & 2 & 6 \\
\hline 9 & - & 2 & - & - & - & 2 & 3 & 9 & 7 & 7 & - & - \\
\hline 10 & - & - & 1 & - & 一 & - & 4 & 12 & 5 & - & 2 & 4 \\
\hline 11 & - & - & - & - & - & - & 2 & 8 & 3 & - & 2 & 8 \\
\hline 12 & - & - & - & - & 2 & - & 2 & 6 & 5 & - & 6 & 8 \\
\hline 13 & - & - & - & - & - & - & 1 & 5 & - & 4 & 5 & 5 \\
\hline 14 & - & - & 1 & 1 & - & - & 10 & 5 & 4 & 1 & 7 & 3 \\
\hline 15 & - & - & - & - & 1 & - & - & 5 & 3 & 3 & $\mathbf{5}$ & 4 \\
\hline 16 & - & 1 & - & - & 1 & 1 & 2 & $j$ & 3 & 1 & - & - \\
\hline 17 & - & - & 1 & - & - & - & 2 & 3 & 2 & 3 & 5 & - \\
\hline 18 & 1 & 1 & - & - & - & 1 & - & 2 & 3 & 9 & - & - \\
\hline 19 & - & - & 1 & 1 & 1 & - & 3 & 3 & 2 & 11 & 3 & 2 \\
\hline 20 & - & 1 & 1 & 1 & - & - & $\mathbf{5}$ & 3 & 3 & 4 & 3 & - \\
\hline 21 & 2 & 2 & -- & - & 1 & 4 & 7 & 5 & 5 & 9 & - & 2 \\
\hline 22 & 3 & - & - & 2 & - & - & 7 & 4 & 3 & 6 & 6 & - \\
\hline 23 & 3 & 2 & - & 4 & - & 2 & 7 & 2 & 3 & 7 & 5 & - \\
\hline 24 & 2 & - & - & 1 & - & 2 & 8 & 6 & 5 & 9 & - & - \\
\hline $2 j$ & - & 2 & - & - & 1 & 3 & 10 & 2 & 3 & 6 & - & - \\
\hline 26 & - & 1 & - & 1 & 3 & - & 10 & 4 & 5 & 8 & 1 & - \\
\hline 27 & 1 & 1 & - & - & - & 4 & 8 & 7 & 3 & 5 & - & 1 \\
\hline 28 & - & 3 & - & 1 & 3 & 2 & 11 & 6 & 4 & 1 & 1 & 1 \\
\hline 29 & 1 & - & 1 & - & 4 & 4 & 9 & 4 & 3 & 4 & - & - \\
\hline 30 & 1 & - & 1 & - & 3 & 5 & 11 & 4 & 5 & 6 & 1 & - \\
\hline 31 & - & - & 3 & - & 4 & - & 11 & 7 & - & 5 & - & 1 \\
\hline
\end{tabular}

Nimnt man Mitteliverthe für jeden Monat, so erhält man Zahlen, welehe angeben, wie viele Radiationspunkte durch scbnittlich in einer Nacht Meteore aussenden. Es sind folgende, denen iclı die jedesmal auftretenden Ausgangspunkte, geordnet nach ihrer jedesmaligen Häufig- keit, beisetze. Leo soll unterstrichen werden, damit man in einem Beispiele leichter den Wechsel der Rangordnung erkenne.

Jan. = 1. 5. Le o. Genini. Cetus. Columba. Pol.

Febr. = 1. 4. Le o. Can. Min. Gemini. Pol. 
März $=1.4$. Leo. Gemini. Pol. Perseus.

April $=1$. 4. L e o. Lyra. Pol. Perseus.

Mai $=2$. 1. Scorpius. Lyra. Pol. Cygnus. Aquila. Le o. Juni $=3$. 3. Sagittarius. Scorpius. Lyra. Pol. Aquila. Cygnus. Aquarius. Caper. Pisc. Austr. Pegasus. L e o.

Juli $=$ 5. 4. Sagittarius. Cassiopea. Caper. Perseus. Aquarius. Cygnus. Aquila. Lyra. Cetus. Pol. Pisc. Austr. Pisces. Draco. Auriga. Le o.

Aug. $=$ 5. 8. Cassiopea. Aquila. Cygnus. Perseus. Aquarius. Lyra. Caper. Sagittarius. Cetus. Pol. Taurus. Draco. Aries. Gemini. Orion. L e o.

Sept. $=4$. 1. Aquarius. Cygnus. Cassiopea. Perseus. Aquila. Cetus. Lyra. Pol. Taúrus. Caper. Sagittarius. Pegasus. Gemini. Leo.
Oct. $=$ 5. 0. Perseus. Cassiopea. Aquarius. Pol. Gemini. Cetus. Leo. Taurus. Auriga. Aries. Cygnus. Can. Mia. Orion. Hydra.

Nov. $=$ 4. 4. Perseus. Cassiopea. Taurus. Le o. Gemini. Pol. Auriga. Aquarius. Aquila. Cetus. Orion. Hydra. Can. Min. Cygnus. Columba. Lyra.

Dec. $=$ 3. 6. Perseus. Gemini. Le o. Pol. Taurus. Auriga. Cassiopea. Hydra. Columbia. Cyguus. Can. Min. Orion. Cetus. Pegasus. Aquarius. Lyra.

So unvollständig nun diese Uebersichten auch sein mügen, so genügen sie doch, vorläufig, um verschiedene bisjetzt fast ganz unbekannte, und nie ernstlich erörterte Verhältnisse der ferneren Erforschung anzuempfehlen.

Athen, 1867 Juni 12. J. F. Julius Schmidt.

\section{Beobachtungen des Cometen II. 1867 auf der Sternw. zu Athen. Von Hern Dir. J. F. Julius Schmidt.}

Nachdem sämmtliche heiter Nächte seit dem Vollmonde des Juli für die Beobachtung des Cometen unbenutzt bleiben mussten, weil abermals mir keine Ephemeride rechtzeitig zukam, glückte die Auffindung des Cometen am 18. August mit Hülfe der Ephemeride von Prof. Bruhns. Der äusserst lichtschwache Nebel hatte noch $2^{\prime}$ Durchmesser, stand an der letzten Gränze der Sichtbarkeit, und kunnte nur mit der grössten Anstrengung beobachtet werden. Aug. 22 und 23 sah ihn wohl. doch war der wolkenlose Himmel rom Staube der Etesien und dem Rauche ferner Waldbrände merklich dunstig. Aug. 24-27 kounte der Comet blickweise noch gesehen werden, aber die Messung war hereits anmöglich. Die den Umständen nach ziemlich guten Positionen sind folgende:

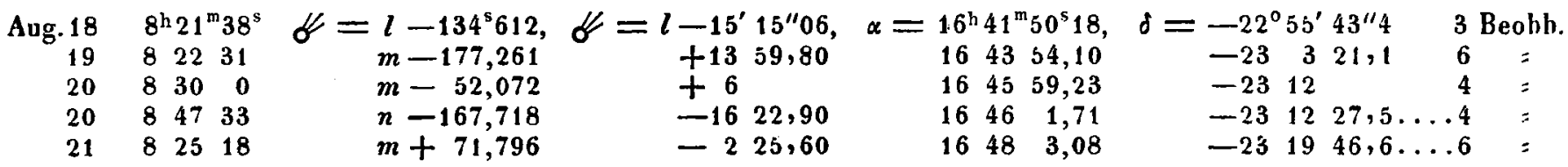

Scheinbarer Ort der Vergleichsterne.

$$
\begin{aligned}
& \text { Aug. } 18 \quad l=16^{\mathrm{h}} 44^{\mathrm{m}} 4^{\mathrm{s}} 80,-22^{\circ} 40^{\prime} 28^{\prime \prime} 4 \\
& 19 m=164651,35 \quad-23 \quad 1720,9 \\
& 20 \quad m=16 \quad 4651,31 \quad-23 \quad 17 \quad 20,9 \\
& 20 \quad n=16 \quad 48 \quad 49,42 \quad-2256 \quad 4,5 \\
& 21 \quad m=164651,29-231721,0 \text {. }
\end{aligned}
$$

Dass die Beobachtungen genau genug sind, um einen letzten Normalort zu bilden, zeigt folgende Vergleichung mit Bruhns' Ephemeride, welche, wie man sieht $1 \frac{1}{2}$ Monate später den Ort noch auf 1' richtig angab. Aus diesem Grunde aber ist eine wesentliche Aenderung der Elemente wohl nicht zu erwarten. Die Parallaxe hahe ich nicht berücksichtigt.
Lal. 30627 .

Lal. 30690, 91. Piazzi 220.

Lal. 30756. Piazxi 234. Argel. - Oeltzen 16125, 26, 27. Rümker 5590. Rob. 3483 .

$$
\begin{aligned}
& \text { Aug. } 18(\mathrm{~B}-\mathrm{B}) \text { in } \alpha=+5^{5} 4 \text {, in } \delta=+0^{\prime} 5 \\
& 19=:=+5,3==+1,1 \\
& 20== \pm+3,8= \pm+0,2 \\
& 21=\quad= \pm+4,7 \quad==+0,2
\end{aligned}
$$

Gebe ich meinen früheren Beobachtungen dieses Cometen das Gewicht $p=4$, so ist für die jetzigen $p=1$ zu setzen.

Athen, 1867 Aug. 29. J. F. Julius Schmidt. 